\title{
Graphite oxide as a support for palladium and rhodium complexes, assessed as catalysts for the partial hydrogenation of I-heptyne
}

\begin{abstract}
Graphite oxide $(\mathrm{GO})$ was used to anchor $\left[\mathrm{PdCl}_{2}(\mathrm{TDA})_{2}\right]$ and $\left[\mathrm{RhCl}(\mathrm{TDA})_{3}\right](\mathrm{TDA}=$ $\left.\mathrm{NH}_{2}\left(\mathrm{CH}_{2}\right)_{12} \mathrm{CH}_{3}\right)$ with $2 \mathrm{wt} \%$ of metal load. Elemental analysis (EA), FTIR, XPS, XRD and atomic absorption spectroscopy (AA) were the techniques employed for characterization. FTIR and XRD revealed that both complexes were mainly immobilized on the GO surface. The partial hydrogenation of 1-heptyne was the test reaction to evaluate the catalytic behavior of these heterogenized complexes under mild conditions of temperature and pressure in a semi continuous process. The results were compared with those obtained with the Wilkinson catalyst under the same operational conditions. AA analysis revealed that there is no leaching of the complexes during the heterogeneous catalytic evaluations. $\left[\mathrm{RhCl}(\mathrm{TDA})_{3}\right] / \mathrm{GO}$ was the best catalyst due to electronic and steric effects as well as the influence of the support.
\end{abstract}

Keywords: graphite oxide, Pd(II) and Rh(I) complexes, Wilkinson catalyst, 1-heptyne, heterogeneous selective hydrogenation

\author{
Volume 4 Issue 4 - 2020 \\ Liprandi DA,' Cagnola EA,' Mastalir A, ${ }^{2}$ \\ Szabó T, ${ }^{3}$ Paredes J,' Martínez Bovier L, ${ }^{4}$ Betti \\ C., ${ }^{4}$ Badano JM, ${ }^{1,4}$ Lederhos CR, ${ }^{4}$ Quiroga \\ $\mathrm{ME}^{1,4}$ \\ 'Departamento de Química, National University of Litoral \\ (UNL), Argentina \\ 2Department of Organic Chemistry, University of Szeged, \\ Hungary \\ ${ }^{3}$ Department of Physical Chemistry and Materials Science, \\ University of Szeged, Hungary \\ ${ }^{4} \mathrm{INCAPE}, \mathrm{CONICET}$, Argentina
}

Correspondence: Quiroga ME, Química Inorgánica, Departamento de Química, INCAPE-CONICET, National University of Litoral (UNL), Santiago del Estero 2829, 3000 Santa Fe, Argentina, Tel +54 34245 I I 370 Int 6109,

Emailmquiroga@fiq.unl.edu.ar

\section{Introduction}

Graphite oxide (GO) is an oxygen-rich carbonaceous material, which may be synthesized by the controlled oxidation of graphite. ${ }^{1,2}$ GO is a two-dimensional solid, with strong covalent bonding within the layers and weaker interlayer contact through intercalated water molecules. ${ }^{1,2}$ The model of Lerf-Klinowski is the most accepted model of $\mathrm{GO},{ }^{3}$ it describes the GO layers as random distributions of flat aromatic regions with unoxidized benzene rings and other regions of alicyclic six-membered rings with tertiary hydroxyl and epoxide groups, but other models in the literature suggest alternative chemical structures. ${ }^{4,5}$ The graphene oxide sheets are terminated with carboxyl groups. ${ }^{3}$ Unlike most lamellar compounds of graphite, GO cannot be characterized by a definite empirical formula, as it is a non-stoichiometric compound and its composition depends on the synthesis conditions. Further, GO is strongly hygroscopic and decomposes above $333 \mathrm{~K} .{ }^{4} \mathrm{GO}$ is also a hydrophilic material, which may be readily dispersed in water to form stable colloidal suspensions. ${ }^{1,2}$ As the GO lamellae are capable of parallel orientation, self-assembled films can be prepared from diluted dispersions. ${ }^{6}$ As a result of liquid sorption, GO readily undergoes swelling and disaggregating. ${ }^{2}$ Moreover, GO has an excellent intercalation ability and cation exchange capacity and therefore a large number of intercalated GO materials have been synthesized and investigated. The intercalation of alkyltrimethylammonium ions resulted in the formation of organophilic GO materials, which have been applied in photochemical reactions. ${ }^{7}$ Organophilic GO materials have also been utilized as the host materials of low-loaded Pd catalysts. ${ }^{8-10}$

Alkene and partial alkyne hydrogenations are closely related to the synthesis and manufacture of food additives, flavors and fragrances, as well as pharmaceutical, agrochemical and petrochemical substances, examples of fine and industrial chemicals. ${ }^{11,12}$ Besides, transition metal complexes are widely used as catalysts for homogeneous and heterogeneous hydrogenation reactions, presenting the advantages of higher activities and selectivity's even under mild conditions. ${ }^{13-17}$ In the last decade, different transition metal complexes of $\mathrm{Co}, \mathrm{Cu}$ and $\mathrm{Rh}$ with different ligands anchored on GO, have been tested for oxidation or reduction reactions. ${ }^{18-21}$ There are few publications on transition metal complexes supported on GO as catalysts for the partial hydrogenation of medium chain terminal alkynes.

The subject of this study is to assess the use of $\left[\mathrm{PdCl}_{2}(\mathrm{TDA})_{2}\right]$ and $\left[\mathrm{RhCl}(\mathrm{TDA})_{3}\right]$, supported on $\mathrm{GO}$, heterogeneous system, as catalysts for the partial hydrogenation of 1-heptyne at mild conditions. The results were compared, under the same operational conditions, with those obtained for:

1) $\left[\mathrm{PdCl}_{2}(\mathrm{TDA})_{2}\right]$ and $\left[\mathrm{RhCl}(\mathrm{TDA})_{3}\right]$ unsupported, homogeneous systems.

2) The Wilkinson catalyst, $\left[\mathrm{RhCl}\left(\mathrm{PPh}_{3}\right)_{3}\right]$, which is considered as a typical reference for partial hydrogenations.

\section{Experimental}

\section{Catalysts preparations}

GO was prepared from a natural graphite (Sigma Aldrich) according to Brodie's method:22 graphite $(99.98 \mathrm{wt} \% \mathrm{C})$ and $\mathrm{NaClO}_{3}$ were mixed in a flask in an ice-bath. Fuming $\mathrm{HNO}_{3}$ was then added drop wise. Another portion of acid was added after a time and the slurry was heated to $333 \mathrm{~K}$ and kept for $8 \mathrm{~h}$. The heating rate $(1.5 \mathrm{Kmin}$ $\left.{ }^{1}\right)$ was controlled to avoid dangerous deflagration. The reaction was 
finished by transferring the mixture into distilled water. Then, the suspension was washed with $\mathrm{HCl}$ solution and with a copious amount of distilled water until the supernatant had a specific conductivity of $10 \mu \mathrm{S} \mathrm{cm}^{-1}$. Finally, the residual graphite oxide was centrifuged and dried.

$\left[\mathrm{PdCl}_{2}(\mathrm{TDA})_{2}\right]$ and $\left[\mathrm{RhCl}(\mathrm{TDA})_{3}\right]$ were synthetized by a procedure previously publishe ${ }^{23}$ by reaction of tridecylamine $\left(\mathrm{TDA}=\mathrm{NH}_{2}\left(\mathrm{CH}_{2}\right)^{12}\right.$ $\mathrm{CH}_{3}$, purity $>98 \%$, Fluka Chemika Catalogue No. 91590) with $\mathrm{PdCl}_{2}$ (Fluka Catalogue No. 76050) or $\mathrm{RhCl}_{3}$ (purity $>98 \%$, Aldrich Catalogue No. 30,786-6) in toluene (Merck, Catalogue No. TX073544) using a glass equipment with agitation and reflux, under an argon atmosphere at $353 \mathrm{~K}$ for $4.5 \mathrm{~h}$. For the $\mathrm{Pd}(\mathrm{II})$ and $\mathrm{Rh}(\mathrm{I})$ complex synthesis molar ratios equal to $\mathrm{TDA} / \mathrm{PdCl}_{2}=2$ and $\mathrm{TDA} / \mathrm{RhCl} 3=6$ were used. Pure complexes, in solid phase, were obtained after the reaction purifying them by a column chromatography and evaporating the solvent in rotavapor equipment. The prepared complexes and the Wilkinson catalyst (purity $>97 \%$, Fluka Catalogue No. 93403) were supported by the incipient wetness technique, ${ }^{24}$ with wt $\%$ of each metal $(\mathrm{M}=\mathrm{Pd}$ or $\mathrm{Rh})$ on the prepared GO previously dried during $2 \mathrm{~h}$ at $333 \mathrm{~K}$. To do so, solutions of all the complexes were prepared using chloroform (purity 99-99.4\%, Merck Catalogue No. 1.02445) as solvent. After impregnation, samples were dried for $1 \mathrm{~h}$ at $333 \mathrm{~K}$ in an oven, and then compressed in discs and divided into pieces of about 2 by $3 \mathrm{~mm}$ approximately. Before each reaction test, the catalysts were dried in an oven during $2 \mathrm{~h}$ at $333 \mathrm{~K}$.

\section{Catalysts characterization}

$\left[\mathrm{PdCl}_{2}(\mathrm{TDA})_{2}\right]$ and $\left[\mathrm{RhCl}(\mathrm{TDA})_{3}\right]$ unsupported (homogeneous systems): Elemental Analysis (EA) and X-ray Photoelectron Spectroscopy (XPS) were used to characterize pure complexes. The former allowed to confirm the presence of $\mathrm{Pd}$ or $\mathrm{Rh}, \mathrm{Cl}$ and $\mathrm{N}$ and their mass fractions as a weight percent on a $\mathrm{C} \& \mathrm{H}$ free base according to standard methods ${ }^{25}$ to determine the stoichiometric ratios of the main atoms and to give a minimum formula for each complex. While XPS technique gave the electronic states of $\mathrm{Pd} 3 \mathrm{~d}_{5 / 2}, \mathrm{Rh} 3 \mathrm{~d}_{5 / 2}, \mathrm{Cl} 2 \mathrm{p}_{3 / 2}$ and $\mathrm{N}$ $1 \mathrm{~s} .{ }^{26,27}$ and superficial atomic ratios of $\mathrm{X} / \mathrm{M}$ (where $\mathrm{M}=\mathrm{Pd}$ or $\mathrm{Rh}$, and $\mathrm{X}=\mathrm{Cl}$ or $\mathrm{N}$ ). EA was carried out via a standard analytical method for each element; and the XPS analysis was performed using a Shimadzu ESCA 750 with an ESCAPAC 760 data acquisition system as already described. ${ }^{26}$

$\left[\mathrm{PdCl}_{2}(\mathrm{TDA})_{2}\right]$ and $\left[\mathrm{RhCl}(\mathrm{TDA})_{3}\right]$ supported on GO(heterogeneous systems): Fourier Transform Infrared spectroscopy (FTIR) and $\mathrm{X}$-Ray Diffraction (XRD) were employed to verify the presence of the complexes and their location on the GO surface respectively. The IR characteristic group wavenumbers of a primary aliphatic amine, in this case TDA, were used as an evidence of the presence of the complexes on the GO after impregnation, ${ }^{28,29}$ that is: $\mathrm{NH}_{2}$ "weak stretching" (3600-3100 $\left.\mathrm{cm}_{-1}\right), \mathrm{CH}$ "very strong stretching" (3000-2800 $\left.\mathrm{cm}_{-1}^{-1}\right)$, $\mathrm{NH}_{2}$ "medium to strong bending" (1700-1600 $\left.\mathrm{cm}_{-1}\right), \mathrm{CH}$ "medium to strong bending" (1500-1300 $\left.\mathrm{cm}_{-1}\right)$ and $\mathrm{CN}$ "medium to weak stretching" (1200-1000 $\left.\left.\mathrm{cm}_{-1}\right)\right)^{23} \mathrm{XRD}$ patterns for GO and the GO supported complexes $\left(\left[\mathrm{PdCl}_{2}(\mathrm{TDA})_{2}\right],\left[\mathrm{RhCl}(\mathrm{TDA})_{3}\right]\right.$ or Wilkinson) were useful to determine the sites where the complexes were anchored on the GO, that is: on its surface or between its layers. IR spectra were obtained by using an IR Prestige- 21 equipment, following a procedure previously described..$^{23}$ The spectra of the anchored complexes were obtained by preparing $\mathrm{KBr}$ disks with a concentration between 0.5 and $1 \%$ to avoid saturated spectra. XRD patterns were obtained by using a Shimadzu XD-D1 XRD powder diffraction equipment, with a radiation $\operatorname{CuK} \alpha(\lambda=1.5405 \AA)$ in the range $5<2 \theta<30^{\circ}$ at a scan speed of $2^{\circ} \min -1 .^{30}$

\section{Catalytic reaction}

Catalytic reactions were carried out by using a 2\% (v/v) 1-heptyne (Fluka, Catalogue No. 51950) in toluene solution in a stainless steel batch reactor. The supported complexes were located, in a basket, between two blades in counter-rotation at $800 \mathrm{rpm}$. The operational conditions were $150 \mathrm{kPa}$ of $\mathrm{H}_{2}$ and $303 \mathrm{~K}$ and the reaction time was $150 \mathrm{~min}$. The reactor was PTFE coated to avoid metal contamination from the reactor. In every case a molar ratio 1-heptyne $/ \mathrm{M}=125(\mathrm{M}=\mathrm{Pd}$ or Rh) was used. Neither external nor internal diffusional limitations were detected. ${ }^{31}$ Reactant and products were analyzed by gas chromatography (GC) using a FID detector and a capillary column HP INNOWax (Polyethylene glycol). During the reactions, the possibility of the supported complex leaching was monitored by analyzing the presence of $\mathrm{M}(\mathrm{Pd}$ or $\mathrm{Rh})$ in each solution at the end of the catalytic evaluations, by atomic absorption spectroscopy.

\section{Results and discussion}

\section{Catalysts characterization}

$\left[\mathrm{PdCl}_{2}(\mathrm{TDA})_{2}\right]$ and $\left[\mathrm{RhCl}(\mathrm{TDA})_{3}\right]$ unsupported (homogeneous systems): Pure $\left[\mathrm{PdCl}_{2}(\mathrm{TDA})_{2}\right]$ and $\left[\mathrm{RhCl}(\mathrm{TDA})_{3}\right]$ were the same as reported before, with identical characterization results. The reader can find a more detailed information of these coordination compounds in published papers. ${ }^{16,17}$ From elemental analysis Pd:Cl:N = 1:2:2 and $\mathrm{Rh}: \mathrm{Cl}: \mathrm{N}=1: 1: 3$ molar ratios can be confirmed. XPS spectra interpretation confirmed the following oxidation states: ca. 1 for Rh, 2 for $\mathrm{Pd}$ and -1 for $\mathrm{Cl}$ and suggest a coordination bond between the nitrogen atom and the metal electrophilic centre through the electron lone pair of the nitrogen atom. ${ }^{26,27}$ Besides, atomic ratios amongst elements indicate the following relationships: Pd:Cl:N=1:2:2 and $\mathrm{Rh}: \mathrm{Cl}: \mathrm{N}=1: 1: 3$. Then, EA and XPS results allowed to conclude that the minimum formulae of the complexes can be expressed as: $\left[\mathrm{PdCl}_{2}(\mathrm{TDA})_{2}\right]$ and $\left[\mathrm{RhCl}(\mathrm{TDA})_{3}\right]$, respectively.

$\left[\mathrm{PdCl}_{2}(\mathrm{TDA})_{2}\right]$ and $\left[\mathrm{RhCl}(\mathrm{TDA})_{3}\right]$ supported on GO (heterogeneous systems): The characteristic bands of a primary amine were observed in the FTIR spectra of pure $\left[\mathrm{PdCl}_{2}(\mathrm{TDA})_{2}\right]$ and $\left[\mathrm{RhCl}(\mathrm{TDA})_{3}\right]{ }^{29} \mathrm{The} \mathrm{CH}$ peak $\left(3000-2800 \mathrm{~cm}_{-1}\right)$, labeled (I), is used as an evidence of the presence of the immobilized complexes on the GO support due to its 'very strong absorbance value'.

On the other hand, Figure 1 shows the IR spectra obtained for: (a) $\mathrm{GO}$, (b) $\left[\mathrm{RhCl}(\mathrm{TDA})_{3}\right] / \mathrm{GO}$ and (c) $\left[\mathrm{PdCl}_{2}(\mathrm{TDA})_{2}\right] / \mathrm{GO}$. Spectra (b) and (c) have the same pattern as the spectrum (a), with the exception in the range of 3000-2800 $\mathrm{cm}_{-1}$ (I) where the $\mathrm{CH}$ stretching from the TDA molecule in the complex coordination sphere appears. This confirms the presence of the complex on the support after the immobilization step. The other signals of lower magnitude are lost, amongst the strong intensity of the GO peaks, on the anchored complexes because of their low loadings $(2 \mathrm{wt} \%)$.

Figure 2 displays the XRD diffractograms for (a) GO, (b) $\left[\mathrm{PdCl}_{2}(\mathrm{TDA})_{2}\right] / \mathrm{GO}$, (c) $\left[\mathrm{RhCl}(\mathrm{TDA})_{3}\right] / \mathrm{GO}$ and (d) Wilkinson/GO. The GO characteristic peak is present at almost the same diffraction angles for all of the four materials $\left(2 \theta=13^{\circ}\right)$. For the $\left[\mathrm{PdCl}_{2}(\mathrm{TDA})_{2}\right]$ and $\left[\mathrm{RhCl}(\mathrm{TDA})_{3}\right]$ systems no additional differences were observed with respect to that of the pure GO, which means that these complexes were not situated between the GO layers or that the amount of intercalated species is below the detection limit. On the other hand, in the case of Wilkinson/GO system there are also reflections at lower diffraction angles. This would suggest that the species, at least partially, are intercalated between the layers of the GO structure. The peak, at lower reflection angles, refers to the swelling of the GO layers due to the voluminous triphenylphosphine ligands. 
By combining this information with that obtained from the FTIR spectra, it can be strongly suggested that the coordination compounds are adsorbed on the support as follows:

I. $\mathrm{Pd}(\mathrm{II})$ and $\mathrm{Rh}(\mathrm{I})$ complexes only on the surface.

II. Wilkinson complex on the surface as well as between the graphite oxide layers.

On the other hand, the results of atomic absorption analysis, for all of the remaining solutions after catalytic reactions, show the absence of any metal trace ( $\mathrm{Pd}$ or $\mathrm{Rh}$ ). This fact proves that there is no leaching during the catalytic runs and that the complex species remain anchored on the support.

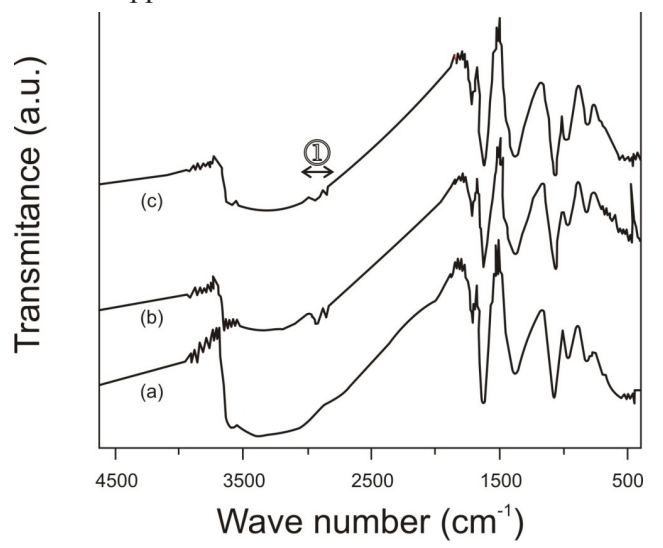

Figure I FTIR spectra for (A) $\mathrm{GO}$, (B) $\left[\mathrm{RhCl}(\mathrm{TDA})_{3}\right] / \mathrm{GO}$ and (C) $\left[\mathrm{PdCl}_{2}(\mathrm{TDA})_{2}\right] / \mathrm{GO}$

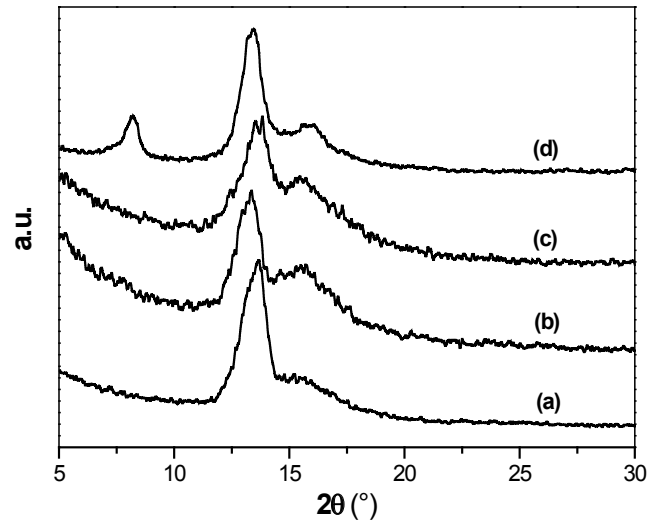

Figure 2 X-ray diffractograms for (a) GO, (b) $\left[\mathrm{PdCl}_{2}(\mathrm{TDA})_{2}\right] / \mathrm{GO}$, (c) $\left[\mathrm{RhCl}(\mathrm{TDA})_{3}\right] / \mathrm{GO}$ and (d) Wilkinson/GO.

\section{Catalytic evaluation}

1-heptene and n-heptane were the only products detected by GC during the hydrogenation reaction. From the chromatographic data were calculated the 1-heptyne Total Conversions $\left(\mathrm{X}_{\mathrm{T}}\right)$, the conversion to 1 -heptene $\left(\mathrm{X}_{1 \text {-heptene }}\right)$ and the Selectivity to 1 -heptene $\left(\mathrm{S}_{1 \text {-heptene }}\right)$ as follow:

$$
\begin{array}{lc}
\mathrm{X}_{\mathrm{T}}(\%)=\left(1-\mathrm{Cy} / \mathrm{Cy}^{\circ}\right) 100 & \text { Eq. (I) } \\
\mathrm{X}_{1-\text { heptene }}(\%)=\left(\mathrm{Ce} / \mathrm{Cy}^{\circ}\right) 100 & \text { Eq. (II) } \\
\mathrm{S}_{1-\text { heptene }}(\%)=\mathrm{Ce} /\left(\mathrm{Cy}^{\circ}-\mathrm{Cy}\right) 100 & \text { Eq. (III) }
\end{array}
$$

$\mathrm{Cy}^{\circ}$ and $\mathrm{Cy}$ are the initial concentration of 1-heptyne and the concentration at a reaction time $t$, respectively $\left[\mathrm{mol} \mathrm{L}^{-1}\right]$. Ce the concentration of 1-heptene at time $\mathrm{t}\left[\mathrm{mol} \mathrm{L}^{-1}\right]$.

The conversions to 1-heptene vs. 1-heptyne Total Conversions $\left(\mathrm{X}_{\mathrm{T}}\right)$ for: a) $\left[\mathrm{PdCl}_{2}(\mathrm{TDA})_{2}\right]$ (homogeneous) and $\left[\mathrm{PdCl}_{2}(\mathrm{TDA})_{2}\right]$
GO (heterogeneous); b) Wilkinson (homogeneous) and Wilkinson/ GO (heterogeneous) and c) $\left[\mathrm{RhCl}(\mathrm{TDA})_{3}\right]$ (homogeneous) and $\left[\mathrm{RhCl}(\mathrm{TDA})_{3}\right] / \mathrm{GO}$ (heterogeneous) are shown in Figure 3 - Figure 5 respectively (Figure 6).

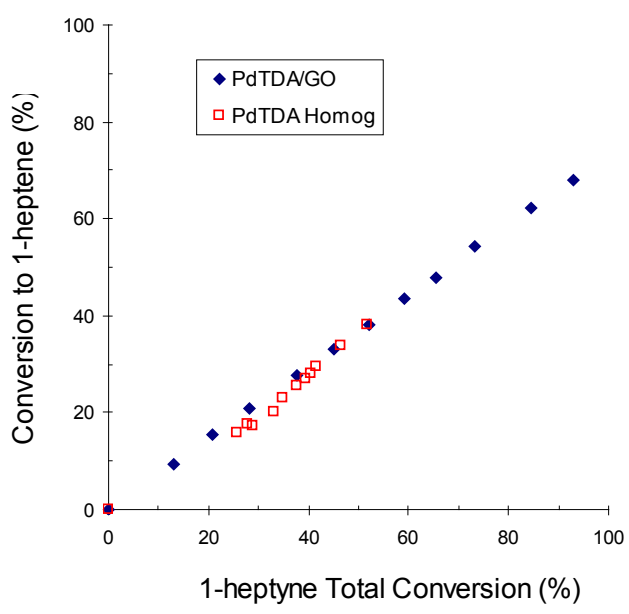

Figure 3 Conversion to I-heptene vs. I-heptyne Total Conversion using $\left[\mathrm{PdCl}_{2}(\mathrm{TDA})_{2}\right](\square)$ or $\left[\mathrm{PdCl}_{2}(\mathrm{TDA})_{2}\right] / \mathrm{GO}(\bullet)$ as catalysts.

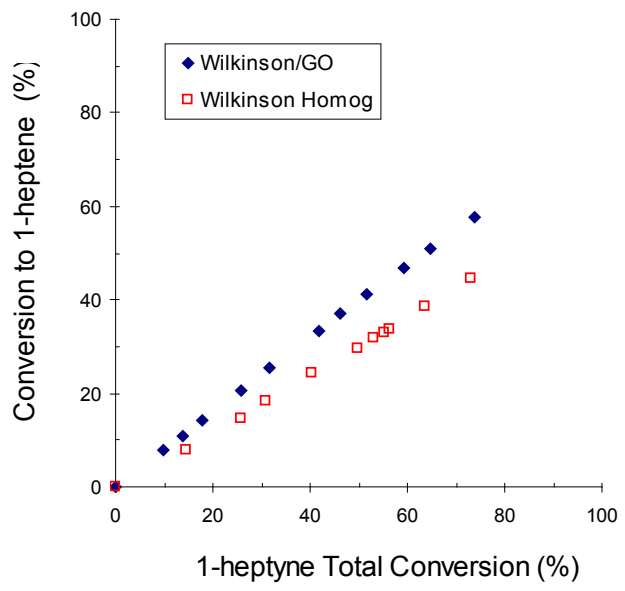

Figure 4 Conversion to I-heptene vs. I-heptyne Total Conversion using Wilkinson $(\square)$ or Wilkinson/GO ( () as catalysts.

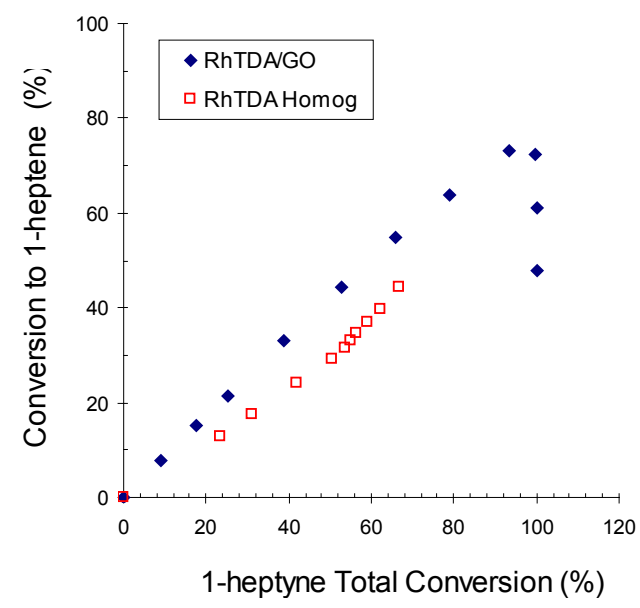

Figure 5 Conversion to I-heptene vs. I-heptyne Total Conversion using $\left[\mathrm{RhCl}(\mathrm{TDA})_{3}\right](\square)$ or $\left[\mathrm{RhCl}(\mathrm{TDA})_{3}\right] / \mathrm{GO}(\downarrow)$ as catalysts. 


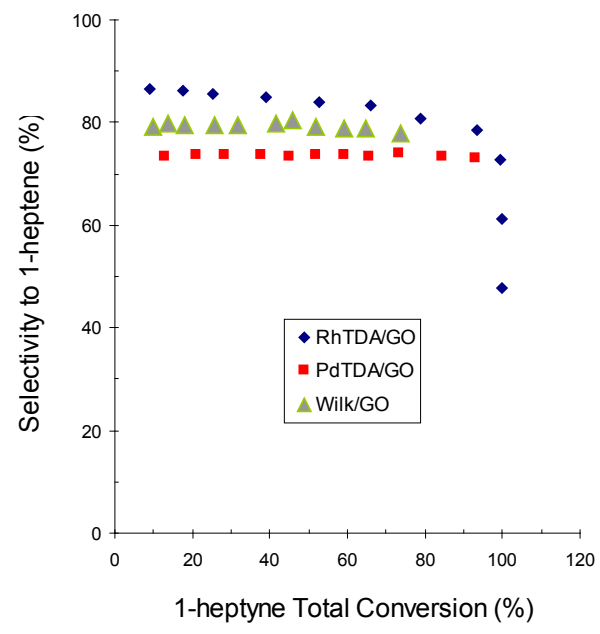

Figure 6 Selectivity to I-heptene vs. I-heptyne total conversion using $\left[\mathrm{RhCl}(\mathrm{TDA})_{3}\right] / \mathrm{GO}(\diamond)$, Wilkinson/GO $(\boldsymbol{\Delta})$ or $\left[\mathrm{PdCl}_{2}(\mathrm{TDA})_{2}\right] / \mathrm{GO}(\boldsymbol{\square})$ as catalysts.

Table 1 exhibits values for 1-heptyne total conversion $\left(\mathrm{X}_{\mathrm{T}}\right)$ and the selectivity to 1-heptene $\left(\mathrm{S}_{1-\text { heptene }}\right)$ for each heterogeneous catalytic system at: (I) $150 \mathrm{~min}$ or (II) 105 min reaction time.

These values can be used to order the complex systems in terms of 1-heptyne total conversion:

Table I I-heptyne total conversion $\left(\mathrm{X}_{\mathrm{T}}\right)$ and selectivity to I-heptene $\left(\mathrm{S}_{\text {I }}\right.$

\begin{tabular}{lll}
\hline \multicolumn{1}{c}{ Catalytic system } & $\mathbf{X}_{\mathbf{T}}(\%)$ & $\mathbf{S}_{\text {I-heptene }}(\%)$ \\
\hline Wilkinson $^{(1)}$ & 72.9 & 61.2 \\
Wilkinson/GO(I) $^{(1)}$ & 73.8 & 78.0 \\
{$\left[\mathrm{PdCl}_{2}(\mathrm{TDA})_{2}\right]^{(1)}$} & 51.9 & 73.0 \\
{$\left[\mathrm{PdCl}_{2}(\mathrm{TDA})_{2}\right] / \mathrm{GO}{ }^{(1)}$} & 93.1 & 73.2 \\
{$\left[\mathrm{RhCl}(\mathrm{TDA})_{3}\right]^{(1)}$} & 67.0 & 63.3 \\
{$\left[\mathrm{RhCl}(\mathrm{TDA})_{3}\right] / \mathrm{GO}^{(1)}$} & 93.3 & 78.4 \\
\hline
\end{tabular}

I. $150 \mathrm{~min}$

II. $105 \mathrm{~min}$

A) Homogeneous Catalytic Systems

Wilkinson $>\left[\mathrm{RhCl}(\mathrm{TDA})_{3}\right]>\left[\mathrm{PdCl}_{2}(\mathrm{TDA})_{2}\right]$

B) Heterogeneous Catalytic Systems

$\left[\mathrm{RhCl}(\mathrm{TDA})_{3}\right] / \mathrm{GO} \cong\left[\mathrm{PdCl}_{2}(\mathrm{TDA}) 2\right] / \mathrm{GO}>>$ Wilkinson/GO

Besides, the order found for the selectivity to 1-heptene for the catalysts was:

C) $\left[\mathrm{RhCl}(\mathrm{TDA})_{3}\right] / \mathrm{GO} \cong$ Wilkinson/GO $>\left[\mathrm{PdCl}_{2}(\mathrm{TDA})_{2}\right] / \mathrm{GO} \cong$ $\left[\mathrm{PdCl}_{2}(\mathrm{TDA})_{2}\right]>\left[\mathrm{RhCl}(\mathrm{TDA})_{3}\right] \cong$ Wilkinson.

The performance of the catalysts can be interpreted in terms of the following items: I) electronic effects, related to the frontier orbitals of the complexes, II) differential concentration influence, due to the presence of the GO support, and III) steric factor, given by the ligands $\left(\mathrm{L}=\mathrm{TDA}\right.$ or $\left.\mathrm{PPh}_{3}\right)$ of the coordination compounds. A conceptual brief description for each of them is given bellow:

\section{I) Electronic effects}

Our complexes are the active sites that facilitate the reaction between $\mathrm{H}_{2}$ and 1-heptyne, during the catalytic cycle, through the valence orbitals of the complexes. These valence orbitals can be typified as HOMO (highest occupied molecular orbital) and LUMO (lowest unoccupied molecular orbital). The former can render electron density to the anti-bonding hydrogen orbital favoring the cleavage of $\mathrm{H}-\mathrm{H}$ bond. This interaction generates hydrogen atoms that are ready to attack the $\mathrm{C}-\mathrm{C}$ multiple bonds which, simultaneously, are debilitated because the LUMO orbital is available to receive electron density from the $\pi$ bonds of the alkyne. In this context, the higher the energy level of the HOMO/LUMO orbitals the easier the catalytic cycle. For complexes, the HOMO/LUMO orbitals are mainly defined, as predicted by the angular overlap model (AOM), ${ }^{32}$ by the atomic orbitals (AO) of the central atom and its oxidation state influences, in an inverse way, their energy levels. Then, this fact favours the $\mathrm{Rh}(\mathrm{I})$ complexes ([RhCl(TDA) $\left.)_{3}\right]$ and Wilkinson) performance because its frontier orbitals lie higher in energy due to its lower oxidation number.

\section{II) Differential concentration influence}

In our heterogeneous systems the presence of the GO support provides a higher concentration of the substrate around the supported complexes than in the bulk solution, due to an adsorption process on its surface, favoring an increase of the reaction rate. Additionally, as at some time 1-heptyne and 1-heptene coexist we note that the alkyne ( $2 \pi$ bonds) is more strongly adsorbed than the alkene ( $1 \pi$ bond). This means a higher differential concentration for 1-heptyne as far as this species is more significant than the alkene in the reactor.

\section{III) Steric factor}

The performance of the coordination compounds, as catalysts, may be influenced by the size of the ligands. This is so as the ligand could act as a "kind of screen", steric factor, between the species involved in the catalytic cycle. For our catalytic systems the main difference between ligands is the presence of the "bulky" triphenylphosphine $\left(\mathrm{PPh}_{3}\right)$ in the Wilkinson complex vs. the "skinny" TDA in $\left[\mathrm{RhCl}(\mathrm{TDA})_{3}\right]_{3}$ and $\left[\mathrm{PdCl}_{2}(\mathrm{TDA})_{2}\right]$ complexes. The steric factor of the former ligand can take relevance when the complex is in the supported condition because of the size of the $\mathrm{PPh}_{3}$, and the presence of the three of them in the Wilkinson coordination sphere, occupies a larger portion of the GO support. Then, the "screening factor" could provoke a lower adsorbed substrate concentration around the Rhodium atom (Item II) implying a poorer catalytic performance for the Wilkinson complex.

On the basis of these items, we can explain the catalytic performance of our systems as follows:

For homogeneous catalysts, a differential concentration of the substrate (Item II) does not exist as the support is absent. Besides, the steric factor (Item III) is not relevant because in the solution the complexes have the freedom of adopting different conformational structures and so "the screening factor" is much less important. In this context, the electronic effect (Item I) is dominant and responsible for the catalysts performances and so the Wilkinson and $\left[\mathrm{RhCl}(\mathrm{TDA})_{3}\right]$ complexes show higher $\mathrm{X}_{\mathrm{T}}$ and lower $\mathrm{S}_{1 \text {-heptene }}$ values. The higher energy frontier orbitals of these species offer a better condition for the alkyne hydrogenation, not only for the desired product (1-heptene) but, from it to the undesired one (n-heptane) as well.

For heterogeneous catalysts Items I), II) and III) must be considered and the interplay amongst them will define the catalytic 
systems behaviour. For all of them a higher substrate concentration (1-heptyne) around the complex (Item II) is present but it is much less important for the Wilkinson complex because of the "screening factor" (Item III). Additionally, $\left[\mathrm{RhCl}(\mathrm{TDA})_{3}\right]$ and the Wilkinson complex offer a better electronic effect (Item I) than $\left[\mathrm{PdCl}_{2}(\mathrm{TDA})_{2}\right]$. Under these arguments, the order shown in series B) can be explained saying that the 1-heptyne total conversion $\left(\mathrm{X}_{\mathrm{T}}\right)$, for the supported complexes is globally defined by item II. But, the electronic effect (item I) marks the slight difference between $\left[\mathrm{RhCl}(\mathrm{TDA})_{3}\right]$ and $\left[\mathrm{PdCl}_{2}(\mathrm{TDA})_{2}\right]$ at equal differential substrate concentration. On the other hand, the order of the selectivity for the heterogeneous catalysts (first half part of series C) is explained by the electronic effect (item I). This is valid as far as the 1-heptyne differential concentration keeps high enough in the reactor. From all of the evaluated catalytic systems, the most active and selective one was $\left[\mathrm{RhCl}(\mathrm{TDA})_{3}\right] / \mathrm{GO}$, which, in turn, presents the following advantages: 1) it provides the highest conversion to 1-heptene in the shortest reaction time, and 2) it is a heterogeneous system, and so the catalyst can be easily recovered from the reaction mixture and reused.

\section{Conclusion}

Graphite oxide (GO) was used as a support material for $\left[\mathrm{RhCl}(\mathrm{TDA})_{3}\right]$ and $\left[\mathrm{PdCl}_{2}(\mathrm{TDA})_{2}\right]$ complexes due to its high adsorption capacity and lamellar structure. Catalysts with $2 \mathrm{wt} \%$ metal loadings ( $\mathrm{Pd}$ or $\mathrm{Rh}$ ) were prepared by immobilization of $\left[\mathrm{PdCl}_{2}(\mathrm{TDA})_{2}\right]$ and $\left[\mathrm{RhCl}(\mathrm{TDA})_{3}\right]$ complexes on $\mathrm{GO}$ by the incipient wetness technique. $\mathrm{XRD}$ and FTIR investigations gave evidence that the adsorption of these species on the support took place mainly on its surface.

The catalytic behavior of the complexes in homogeneous or heterogeneous systems was studied for the partial hydrogenation of 1 -heptyne in a toluene solution. The Wilkinson complex was used as a reference under the same operational conditions. The finding that the $\left[\mathrm{RhCl}(\mathrm{TDA})_{3}\right] / \mathrm{GO}$ was the best catalytic system, may be interpreted by considering electronic effects, influence of the support and steric factor.

The fact that the best catalytic system was a heterogeneous catalyst has extra benefits such as easy recovery and reuse. Furthermore, as the solvent leaching effect is absent there is no complex migration from the support to the solution and so the desired product (1-heptene) is not poisoned by heavy metals and an extra purification step is not needed.

\section{Acknowledgments}

Financial support obtained from ANPCyT, UNL, CONICET and MINCyT of Argentina, and Project No. 124851, provided by the National Research, Development and Innovation Fund of Hungary, financed under the FK funding scheme, are gratefully acknowledged. This research was conducted in the frame of an Argentine-Hungarian Intergovernmental Science \& Technology cooperation.

\section{Conflicts of interest}

Authors declare that there is no conflict of interest.

\section{References}

1. Stankovich S, Piner RD, Nguyen ST, et al. Synthesis and exfoliation of isocyanate-treated graphene oxide nanoplatelets. Carbon. 2006;44:3342.

2. Dékány I, Krüger-Grasser R, Weiss A. Selective liquid sorption properties of hydrophobized graphite oxide nanostructures. Colloid Polym Sci. 1998;276:570.
3. He H, Riedl T, Lerf A, et al. Solid-State NMR Studies of the Structure of Graphite Oxide. J Phys Chem. 1996;100:19954.

4. Szabó T, Berkesi O, Forgó P, et al. Evolution of surface functional groups in a series of progressively oxidized graphite oxides. Chem Mater. 2006;18:2740.

5. Dreyer DR, Park S, Bielawski CW, et al. Chem Soc Rev. 2010;39:228

6. Kotov NA, Dékány I, Fendler JH. J Phys Chem. 1996;99:13065.

7. Matsuo Y, Miyabe T, Fukutsuka T, et al. Carbon. 20017;45:1005

8. Mastalir Á, Király Z, Benkő M, et al. Catal Lett. 2008;124:34.

9. Mastalir Á, Király Z, Patzkó Á, et al. Synthesis and catalytic application of Pd nanoparticles in graphite oxide. Carbon. 2008;46:1631.

10. Mastalir Á, Szabó T, Király Z, et al. Synthesis and catalytic investigation of organophilic Pd/graphite oxide nanocomposites. Catal Commun. 2012;17:104.

11. Chinchilla R, Nájera C. Chemicals from Alkynes with Palladium Catalysts. Chem Rev. 2014;114:1783.

12. Oger C, Balas L, Durand T, et al. Are Alkyne Reductions Chemo-, Regio-, and Stereoselective Enough To Provide Pure (Z)-Olefins in Polyfunctionalized Bioactive Molecules? Chem Rev. 2013;113:1313.

13. Park JW, Chung YM, Suh YW, et al. Partial hydrogenation of 1,3-cyclooctadiene catalyzed by palladium-complex catalysts immobilized on silica. Catal Today. 2004;3-95:445.

14. Lemus-Yegres LJ, Román-Martínez MC, Such-Basáñez I, et al. Effects of confinement in hybrid diamine-Rh complex-carbon catalysts used for hydrogenation reactions. Microporous and Mesoporous Materials. 2008;109:305.

15. Zgolicz PD, Cabrera MI, Grau RJ. Kinetics of the homogeneous hydrogenation of avermectins catalyzed by $\mathrm{RhCl}(\mathrm{Ph} 3 \mathrm{P}) 3$ complexes. App Catal A: Gen. 2005;283:99.

16. Liprandi D, Cagnola E, Lederhos C, et al. Hydrogenation I. Karamé. Rijeka, Croatia. InTech; 2012.

17. Liprandi D, Cagnola E, Paredes J, et al. A High $(\mathrm{Z}) /(\mathrm{E})$ Ratio Obtained During the 3-Hexyne Hydrogenation with a Catalyst Based on a $\mathrm{Rh}(\mathrm{I})$ Complex Anchored on a Carbonaceous Support. Catal Let. 2012;142(2):231.

18. Dongil AB, Bachiller-Baeza B, Guerrero-Ruiz A, et al. Chemoselective hydrogenation of cinnamaldehyde: A comparison of the immobilization of Ru-phosphine complex on graphite oxide and on graphitic surfaces. $J$ Catal. 2011;282:299.

19. Zhao Q, Chen D, Li Y, et al. Nanoscale. 2013;5:882.

20. Dongil AB, Bachiller-Baeza B, Guerrero-Ruiz A, et al. Graphite oxide as support for the immobilization of Ru-BINAP: Application in the enantioselective hydrogenation of methylacetoacetate. Catal Comm. 2012;26:149.

21. Li Z, Wu S, Ding H, et al. New J Chem. 2013;37:1561.

22. Brodie BC. Ann Chim Phys. 1860;59:466.

23. Cagnola EA, Quiroga ME, Liprandi DA, et al. Immobilized Rh, Ru, Pd and Ni complexes as catalysts in the hydrogenation of cyclohexene. Appl Catal A: Gen. 2004;274:205.

24. Seoane XL, L'Argentière PC, Fígoli NS, et al. Cat Lett. 1992;16:129.

25. Anderson S, Basolo F. Inorg Synth. 1963;7:214.

26. Wagner CD, Riggs WM, Davis LE, et al. Handbook of X-ray photoelectron spectroscopy. In: Mullenberg GE, editor. Eden Preirie, USA. PerkinElmer Corporation. 
27. NIST. X-ray Photoelectron Spectroscopy Database NIST Standard Reference Database 20, Version 4.1 (Web Version:). USA. National Institute of Standards and Technology; 2012.

28. Pouchert CJ. The Aldrich Library of Infrared Spectra. 3rd edn. Wisconsin, USA. Aldrich Chemical Company Inc; 1981:163-164.

29. Silverstein RM. Clayton-Basler G, Morril TC. Spectrometric Identification of Organic Compounds. 5th edn. New York, USA. John Wiley \& Sons Inc; 1991:123-124.
30. Cobo M, Quintero A, Montes de Correa C. Liquid phase dioxin hydrodechlorination over $\mathrm{Pd} / \gamma-\mathrm{Al}_{2} \mathrm{O}_{3}$. Catal Today. 2008;133:509.

31. Holland FA, Chapman FS. Liquid Mixing and Processing in Stirred Tanks. Reinhold, New York, USA. 1976.

32. Purcell KF, Kotz JC. Inorganic Chemistry, Holt-Saunders International Editions. Philadelphia. 1977:543-549. 